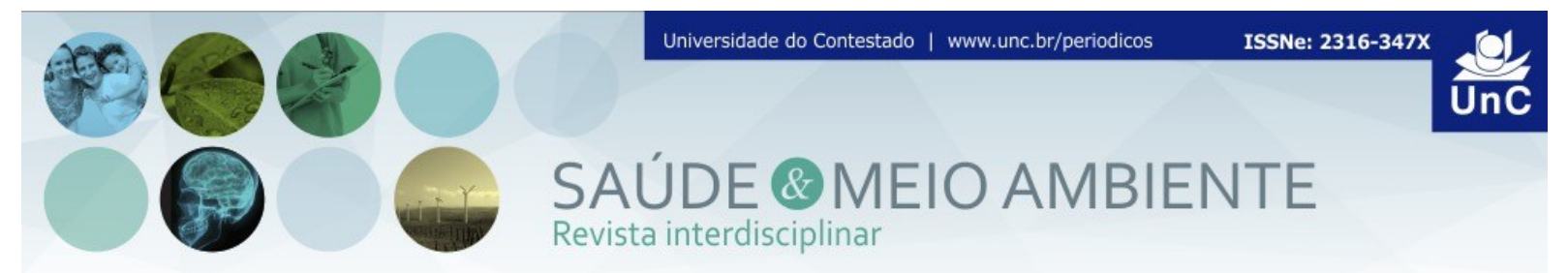

\title{
CONTRIBUIÇÃO AO CONHECIMENTO DA ORNITOFAUNA DO MUNICÍPIO DE JOINVILLE, SANTA CATARINA, BRASIL
}

Luciano Souza Costa ${ }^{1}$

\begin{abstract}
RESUMO: Este trabalho teve como objetivo realizar um levantamento qualitativo da ornitofauna do município de Joinville, localizado na região norte do estado de Santa Catarina. Foram efetivados 26 levantamentos de campo entre Junho de 2012 a novembro de 2014, utilizando técnicas de levantamentos já consagradas em levantamentos ornitológicos: contato visual, busca de vestígios, rede de neblina, identificação de vocalizações e playback. Durante este período foram inventariadas 344 espécies da ornitofauna (187 passeriformes e 157 não passeriformes), distribuídas em 24 ordens e 70 famílias. Das espécies registradas 31 estão ameaçadas de extinção para Santa Catarina e 18 são aves migratórias. Os resultados obtidos revelam a importância do município para a conservação da ornitofana, devido à grande diversidade de espécies de aves.
\end{abstract}

Palavras-chaves: Ornitofauna. Inventário qualitative. Joinville.

\section{CONTRIBUTION TO THE KNOWLEDGE OF THE MUNICIPALITY OF JOINVILLE BIRDLIFE, SANTA CATARINA, BRAZIL}

ABSTRACT: This study aimed to conduct a qualitative survey of birdlife the city of Joinville, located in the northern region of the state of Santa Catarina. Were hired 26 field surveys from June 2012 to November 2014, using survey techniques already established in ornithological surveys: eye contact, search for traces, Fog network, vocalizations identification and playback. During this period were raised 344 species of birdlife (187 and 157 not passerines passerines), distributed in 24 orders and 70 families. Of the species recorded 31 are endangered to Santa Catarina and 18 are migratory birds. The results show the importance of the city for the conservation of birdlife due to the great diversity of bird species.

Key words: birdlife. Qualitative inventory. Joinville.

\footnotetext{
${ }^{1}$ Biólogo, MSc, Universidade Federal de Santa Catarina. Zoologia-avifauna. Santa Catarina. Brasil. Email: luciano.viva@gmail.com
} 


\section{INTRODUÇÃO}

O Brasil abriga uma das maiores diversidades da avifauna mundial, com 1.901 espécies de aves, abrangendo 33 ordens e 103 famílias (CBRO, 2014). Este montante corresponde a mais da metade das espécies de aves registradas para a América do Sul $(n=2.950)$ e aproximadamente $18 \%$ de toda a riqueza de aves do mundo ( $n=10.488)$ (WEIMER et al., 2014), colocando o Brasil entre os três países com maior riqueza de aves juntamente com Colômbia e Peru. Das espécies registradas para o território brasileiro, 891são assinaladas para a Mata Atlântica e ecossistemas associados, sendo que destas 199 são consideradas endêmicas (MOREIRA- LIMA, 2013).

O estado de Santa Catarina encontra-se totalmente inserido no bioma Mata Atlântica, estando entre os três estados com melhor grau de conservação deste bioma. Apesar disto, às constantes intervenções antrópicas em áreas naturais vem contribuindo para elevar o número de espécies ameaçadas de extinção. Atualmente - estado catarinense aponta 34 espécies de aves incluídas na lista da fauna brasileira de espécies ameaçadas de extinção (IBAMA, 2003). Segundo a lista da fauna ameaçada para o Estado de Santa Catarina (CONSEMA, 2011), são 97 espécies de aves ameaçadas, sendo 28 na categoria de criticamente em perigo, 31 em perigo e 38 vulnerável. É importante ressaltar que o acréscimo no número de espécies ameaçadas de extinção não somente está relacionado como as intervenções antrópicas, mas também, pelo aumento no nível de conhecimento ornitológico, causado pelos inúmeros levantamentos realizados no estado nos últimos anos (GHIZONI-JR, 2004; AZEVEDO, GHIZONI-JR, 2005; GHIZONI-JR; SILVA, 2006; AMORIM; PIACENTINI, 2006; RUPP et al., 2008; GHIZONI et al., 2009; GROSE, 2011), possibilitando estabelecer um diagnóstico mais preciso de cada espécie.

No entanto, apesar das várias investigações realizadas no âmbito estadual, pouco se sabe sobre a riqueza ornitológica do município de Joinville. Dentre as poucas publicações científicas podem ser mencionadas (SEVEGNANI et al., 2009) que estudou a avifauna do Jardim Botânico da Universidade Regional de Joinville e seu entorno; (CREMER, 2010; GROSE et al., 2011), focaram suas pesquisas nas aves estuarinas da Baía da Babitonga e (GROSE, 2013), realizou levantamento ornitológico em três unidades de conservação do município.

Nesse sentido, o presente estudo teve por objetivo apresentar os resultados dos 26 levantamentos qualitativos realizados no município de Joinville, no período entre 2012 a 2014 e, assim, contribuir com mais informações sobre sua ornitofauna. 


\section{MATERIAIS E MÉTODOS}

\section{ÁREA DE ESTUDO}

O estudo foi realizado no município de Joinville, localizado na região norte do estado de Santa Catarina, entre as coordenadas geográficas de $26^{\circ} 18^{\prime} 18^{\prime \prime}$ Sul e $48^{\circ} 50^{\prime} 46 "$ Oeste. Com uma área de $1.126,106 \mathrm{~km}^{2}$, é considerada a maior cidade do estado, com uma população de 554,601 habitantes (IBGE, 2014). Sua vegetação faz parte do Bioma Mata Atlântica, estando representado na área de estudo pelas formações de Floresta Ombrófila floresta e Floresta Ombrófila Densa. Esta última formação assume tipologias diferentes de acordo com sua altitude. No município estão presentes as subformações de terras baixas, submontana, montana e altomontana. Ocorre também formação pioneira flúvio-marinho (manguezal) e campos de altitude, além de outros ecossistemas associados como lagoa, baia e rios entre outros. A hidrografia apresenta-se predominantemente na vertente Atlântica da Serra do Mar, cujos rios se caracterizam por pequena extensão e grande vazão. Conforme classificação de Köppen, o clima de Joinville é o Cfa, que significa "clima mesotérmico, úmido, sem estação seca". A precipitação média mensal varia entre 100 e $350 \mathrm{~mm}$ e a umidade relativa pode atingir até $85 \%$ e a temperatura média anual nos doze últimos anos é de $22,63^{\circ} \mathrm{C}$, sendo a média das máximas $27,18^{\circ} \mathrm{C}$ e a média as mínimas de $18,91^{\circ} \mathrm{C}$.

\section{METODOLOGIA}

Entre junho de 2012 a novembro de 2014, foram realizados diversos levantamentos ornitológicos, necessários para obtenção de licenças ambientais para instalação e operação de empreendimentos no município, assim como para estudos de viabilidades, monitoramentos ambientais, planos de manejos e pesquisas.

Em cada levantamento realizado no município, os dados coligados em campo eram tabulados para uma planilha, formando um banco de dado por trabalho. Em uma segunda etapa, todos os dados obtidos nestes levantamentos foram compilados para uma única planilha, constituindo um banco de dados com todas as espécies inventariadas no município, durante o período estabelecido.

Os levantamentos foram realizados através de técnicas de campo convencionais para estudos ornitológicos qualitativos e quantitativos, tais como: contato visual com auxílio de binóculos, busca de vestígios, rede de neblina, identificação de vocalizações e playback. Em grande parte dos levantamentos os horários estabelecidos foram as primeiras horas do dia (6:00 às 10:00 horas), período de maior atividade das aves. Em algumas ocasiões também foram realizados visitas ao entardecer e noturnas, objetivando visualizar aves com hábitos crepusculares e noturnos. 
No total foram realizados 26 levantamentos, abrangendo ecossistemas de floresta ombrófila mista, floresta ombrófila densa nas subformação de terras baixas, submontana, montana e altomontana, em vários estágios sucessionais, assim como formações pioneiras flúvio-marinho (manguezal), ambientes aquáticos e áreas urbanizadas, entre outros. Além dos levantamentos sistêmicos, também foram acrescentadas ao banco de dados informações obtidas de forma não sistemática.

Entre os guias e livros usados nas identificações estão (SICK, 1997; SOUZA, 2004, SIGRIST, 2006). A classificação taxonômica seguiu a estipulada pelo Comitê Brasileiro de Registros Ornitológicos, 2014. O status de espécies ameaçadas seguiu a Lista da Fauna Ameaçada de Extinção para o Estado de Santa Catarina (CONSEMA, 2011). Já para as aves migratórias, utilizou-se a lista das espécies de aves migratórias ocorrentes no Brasil (CEMAVE, 2005).

\section{RESULTADOS E DISCUSSÃO}

Foram registradas para o município de Joinville, um total de 344 espécies de aves, distribuídas em 70 famílias e 24 ordens, correspondendo $18 \%$ das aves brasileiras e $58 \%$ das aves catarinenses. Deste montante, 187 são da ordem dos passeriformes e 157 dos não passeriformes, conforme descrito no quadro 1.

Essa considerável riqueza deve-se principalmente pelo esforço amostral empregado, assim como pela grande diversidade de hábitats e micro hábitats, decorrente da heterogeneidade fitofisionômicas ao longo da gradação altitudinal da floresta ombrófila densa, bem como dos vários estágios sucessionas que elas apresentam. A presença da floresta ombrófila mista, campos de altitudes e outros ecossistemas associados também contribuem para vultosa riqueza da avifauna do município.

Ghizoni-Jr et al., (2013), em levantamento realizado na llha de Santa Catarina, registrou a ocorrência de 352 espécies, semelhante aos resultados obtidos neste estudo. GROSE, (2013), realizou levantamentos em três unidades de conservação no município de Joinville, registrando a ocorrência de 241 espécies, aquém do obtido neste estudo.

As famílias mais representativas em número de espécies foram Thraupidae $(n=34)$ e Tyrannidae $(n=28)$, padrão também observado por (WEIMER et al., 2014), em inventário realizado em um fragmento florestal no centro urbano do município de Xanxerê, onde estas duas famílias apresentaram o maior número de espécies.

A família Thraupidae é restrita à América, sendo que $60 \%$ delas vivem na América do Sul e destas, $30 \%$ vivem nos Andes. Estas aves agrupam-se geralmente em pequenos bandos de três a cinco indivíduos. No que diz respeito família Tyrannidae, grande parte dos representantes são migrantes intracontinentais, representando $33,5 \%$ das aves que realizam este tipo de deslocamento (NUNES, 2008). 
Tal predominância das famílias Thraupidae e Tyrannidae pode-se justificar pelo fato de apresentarem espécies conspícuas, sendo facilmente observadas.

Quadro 1 - Relação das espécies de aves registradas para o município de Joinville - SC, nome do táxon, nome em português, aves migratórias, status e obtenção das informações.

\begin{tabular}{|c|c|c|c|}
\hline Nome do Táxon & Nome em Português & Aves Migratórias & Status \\
\hline \multicolumn{4}{|l|}{ TINAMIFORMES } \\
\hline \multicolumn{4}{|l|}{ Tinamidae } \\
\hline Tinamus solitarius & Macuco & & VU \\
\hline Crypturellus obsoletus & inhambuguaçu & & \\
\hline Crypturellus tataupa & inhambu-chintã & & \\
\hline Crypturellus noctivagus & jaó-do-sul & & EN \\
\hline \multicolumn{4}{|l|}{ ANSERIFORMES } \\
\hline \multicolumn{4}{|l|}{ Anatidae } \\
\hline Dendrocygna bicolor & marreca-caneleira & & \\
\hline Dendrocygna viduata & irerê & & \\
\hline Cairina moschata & pato-do-mato & & \\
\hline Amazonetta brasiliensis & pé-vermelho & & \\
\hline Anas flavirostris & marreca-pardinha & & \\
\hline Anas bahamensis & marreca-toicinho & & \\
\hline Anas versicolor & marreca-cricri & & \\
\hline \multicolumn{4}{|l|}{ GALLIFORMES } \\
\hline \multicolumn{4}{|l|}{ Cracidae } \\
\hline Ortalis squamata & Aracuã-escamoso & & \\
\hline Penelope superciliaris & jacupemba & & VU \\
\hline Penelope obscura & jacuaçu & & \\
\hline Aburria jacutinga & jacutinga & & CR \\
\hline \multicolumn{4}{|l|}{ Odontophoridae } \\
\hline Odontophorus capueira & uru & & \\
\hline \multicolumn{4}{|l|}{ PODICIPEDIFORMES } \\
\hline \multicolumn{4}{|l|}{ Podicipedidae } \\
\hline Rollandia rolland & mergulhão-de-orelha-branca & & \\
\hline Podilymbus podiceps & mergulhão-caçador & & \\
\hline \multicolumn{4}{|l|}{ SPHENISCIFORMES } \\
\hline \multicolumn{4}{|l|}{ Spheniscidae } \\
\hline Spheniscus magellanicus & pinguim-de-magalhães & VS & \\
\hline \multicolumn{4}{|l|}{ SULIFORMES } \\
\hline \multicolumn{4}{|l|}{ Fregatidae } \\
\hline Fregata magnificens & tesourão & & \\
\hline \multicolumn{4}{|l|}{ Sulidae } \\
\hline Sula leucogaster & atobá-pardo & & \\
\hline \multicolumn{4}{|l|}{ Phalacrocoracidae } \\
\hline Phalacrocorax brasilianus & biguá & & \\
\hline \multicolumn{4}{|l|}{ PELECANIFORMES } \\
\hline \multicolumn{4}{|l|}{ Ardeidae } \\
\hline Nycticorax nycticorax & savacu & & \\
\hline Nyctanassa violacea & savacu-de-coroa & & \\
\hline Butorides striata & socozinho & & \\
\hline
\end{tabular}




\begin{tabular}{|c|c|c|c|}
\hline Bubulcus ibis & garça-vaqueira & & \\
\hline Ardea cocoi & garça-moura & & \\
\hline Ardea alba & garça-branca-grande & & \\
\hline Syrigma sibilatrix & maria-faceira & & \\
\hline Egretta caerulea & garça-azul & & \\
\hline Egretta thula & garça-branca-pequena & & \\
\hline \multicolumn{4}{|l|}{ Threskiornithidae } \\
\hline Eudocimus ruber & guará & & CR \\
\hline Plegadis chihi & caraúna-de-cara-branca & & \\
\hline Phimosus infuscatus & tapicuru-de-cara-pelada & & \\
\hline Theristicus caudatus & curicaca & & \\
\hline Platalea ajaja & colhereiro & & \\
\hline \multicolumn{4}{|l|}{ CATHARTIFORMES } \\
\hline \multicolumn{4}{|l|}{ Cathartidae } \\
\hline Cathartes aura & \multicolumn{2}{|l|}{ urubu-de-cabeça-vermelha } & \\
\hline Coragyps atratus & urubu-de-cabeça-preta & & \\
\hline \multicolumn{4}{|l|}{ ACCIPITRIFORMES } \\
\hline \multicolumn{4}{|l|}{ Pandionidae } \\
\hline Pandion haliaetus & águia-pescadora & VN & \\
\hline \multicolumn{4}{|l|}{ Accipitridae } \\
\hline Elanoides forficatus & gavião-tesoura & VN & \\
\hline Harpagus diodon & gavião-bombachinha & & \\
\hline Accipiter superciliosus & gavião-miudinho & & VU \\
\hline Accipiter striatus & gavião-miúdo & & \\
\hline Rostrhamus sociabilis & gavião-caramujeiro & & \\
\hline Heterospizias meridionalis & gavião-caboclo & & \\
\hline Amadonastur lacernulatus & gavião-pombo-pequeno & & VU \\
\hline Urubitinga urubitinga & gavião-preto & & \\
\hline Rupornis magnirostris & gavião-carijó & & \\
\hline Geranoaetus albicaudatus & gavião-de-rabo-branco & & \\
\hline Pseudastur polionotus & gavião-pombo-grande & & \\
\hline Buteo brachyurus & gavião-de-cauda-curta & & \\
\hline \multicolumn{4}{|l|}{ FALCONIFORMES } \\
\hline \multicolumn{4}{|l|}{ Falconidae } \\
\hline Caracara plancus & caracará & & \\
\hline Milvago chimachima & carrapateiro & & \\
\hline Milvago chimango & chimango & & \\
\hline Herpetotheres cachinnans & acauã & & \\
\hline Micrastur ruficollis & falcão-caburé & & \\
\hline Micrastur semitorquatus & falcão-relógio & & \\
\hline Falco sparverius & quiriquiri & & \\
\hline \multicolumn{4}{|l|}{ GRUIFORMES } \\
\hline \multicolumn{4}{|l|}{ Aramidae } \\
\hline Aramus guarauna & carão & & \\
\hline \multicolumn{4}{|l|}{ Rallidae } \\
\hline Rallus longirostris & saracura-matraca & & VU \\
\hline Aramides mangle & saracura-do-mangue & & \\
\hline Aramides cajanea & saracura-três-potes & & \\
\hline Aramides saracura & saracura-do-mato & & \\
\hline Amaurolimnas concolor & saracura-lisa & & \\
\hline Porzana albicollis & sanã-carijó & & \\
\hline
\end{tabular}




\begin{tabular}{|c|c|c|c|}
\hline Gallinula galeata & frango-d'água-comum & & \\
\hline Porphyrio martinica & frango-d'água-azul & & \\
\hline Fulica leucoptera & carqueja-de-bico-amarelo & & \\
\hline \multicolumn{4}{|l|}{ CHARADRIIFORMES } \\
\hline \multicolumn{4}{|l|}{ Charadriidae } \\
\hline Vanellus chilensis & quero-quero & & \\
\hline Pluvialis dominica & batuiruçu & VN & \\
\hline Charadrius semipalmatus & batuíra-de-bando & VN & \\
\hline Charadrius collaris & batuíra-de-coleira & VS & \\
\hline \multicolumn{4}{|l|}{ Haematopodidae } \\
\hline Haematopus palliatus & piru-piru & & \\
\hline \multicolumn{4}{|l|}{ Recurvirostridae } \\
\hline Himantopus melanurus & pernilongo-de-costas-brancas & $\mathrm{VN}$ & \\
\hline \multicolumn{4}{|l|}{ Scolopacidae } \\
\hline Gallinago paraguaiae & narceja & & \\
\hline Numenius phaeopus & maçarico-galego & VN & \\
\hline Actitis macularius & maçarico-pintado & VN & \\
\hline Tringa solitaria & maçarico-solitário & VN & \\
\hline Tringa melanoleuca & maçarico-grande-de-perna-amarela & VN & \\
\hline Tringa semipalmata & maçarico-de-asa-branca & VN & \\
\hline Tringa flavipes & maçarico-de-perna-amarela & VN & \\
\hline Calidris canutus & maçarico-de-papo-vermelho & $\mathrm{VN}$ & \\
\hline Calidris alba & maçarico-branco & VN & \\
\hline Calidris fuscicollis & maçarico-de-sobre-branco & VN & \\
\hline \multicolumn{4}{|l|}{ Jacanidae } \\
\hline Jacana jacana & jaçanã & & \\
\hline \multicolumn{4}{|l|}{ Laridae } \\
\hline $\begin{array}{l}\text { Chroicocephalus } \\
\text { maculipennis }\end{array}$ & gaivota-maria-velha & & \\
\hline $\begin{array}{l}\text { Chroicocephalus } \\
\text { cirrocephalus }\end{array}$ & gaivota-de-cabeça-cinza & & \\
\hline Larus dominicanus & gaivotão & & \\
\hline \multicolumn{4}{|l|}{ Sternidae } \\
\hline Sterna hirundinacea & trinta-réis-de-bico-vermelho & & \\
\hline Sterna trudeaui & trinta-réis-de-coroa-branca & & \\
\hline Thalasseus acuflavidus & trinta-réis-de-bando & & \\
\hline \multicolumn{4}{|l|}{ Rynchopidae } \\
\hline Rynchops niger & talha-mar & & \\
\hline \multicolumn{4}{|l|}{ COLUMBIFORMES } \\
\hline \multicolumn{4}{|l|}{ Columbidae } \\
\hline Columbina talpacoti & rolinha-roxa & & \\
\hline Columba livia & pombo-doméstico & & \\
\hline Patagioenas picazuro & pombão & & \\
\hline Patagioenas cayennensis & pomba-galega & & \\
\hline Patagioenas plumbea & pomba-amargosa & & \\
\hline Zenaida auriculata & pomba-de-bando & & \\
\hline Leptotila verreauxi & juriti-pupu & & \\
\hline Geotrygon montana & pariri & & \\
\hline \multicolumn{4}{|l|}{ PSITTACIFORMES } \\
\hline \multicolumn{4}{|l|}{ Psittacidae } \\
\hline Pyrrhura frontalis & tiriba-de-testa-vermelha & & \\
\hline
\end{tabular}

Saúde Meio Ambient. v. 4, n. 2, p. 16-31, jul./dez. 2015

ISSN 2316-347X 


\begin{tabular}{|c|c|c|}
\hline Forpus xanthopterygius & tuim & \\
\hline Brotogeris tirica & periquito-rico & \\
\hline Pionopsitta pileata & cuiú-cuiú & \\
\hline Pionus maximiliani & maitaca-verde & \\
\hline Amazona vinacea & papagaio-de-peito-roxo & EN \\
\hline Triclaria malachitacea & sabiá-cica & VU \\
\hline \multicolumn{3}{|l|}{ CUCULIFORMES } \\
\hline \multicolumn{3}{|l|}{ Cuculidae } \\
\hline Piaya cayana & alma-de-gato & \\
\hline Crotophaga ani & anu-preto & \\
\hline Guira guira & anu-branco & \\
\hline Tapera naevia & saci & \\
\hline \multicolumn{3}{|l|}{ STRIGIFORMES } \\
\hline \multicolumn{3}{|l|}{ Tytonidae } \\
\hline Tyto furcata & coruja-da-igreja & \\
\hline \multicolumn{3}{|l|}{ Strigidae } \\
\hline Pulsatrix koeniswaldiana & murucututu-de-barriga-amarela & \\
\hline Megascops choliba & corujinha-do-mato & \\
\hline Strix virgata & coruja-do-mato & \\
\hline Athene cunicularia & coruja-buraqueira & \\
\hline Asio clamator & coruja-orelhuda & \\
\hline \multicolumn{3}{|l|}{ NYCTIBIIFORMES } \\
\hline \multicolumn{3}{|l|}{ Nyctibiidae } \\
\hline Nyctibius griseus & mãe-da-lua & \\
\hline \multicolumn{3}{|l|}{ CAPRIMULGIFORMES } \\
\hline \multicolumn{3}{|l|}{ Caprimulgidae } \\
\hline Nyctiphrynus ocellatus & bacurau-ocelado & \\
\hline Lurocalis semitorquatus & tuju & \\
\hline Hydropsalis anomala & curiango-do-banhado & EN \\
\hline \multicolumn{3}{|l|}{ APODIFORMES } \\
\hline \multicolumn{3}{|l|}{ Apodidae } \\
\hline Cypseloides fumigatus & taperuçu-preto & \\
\hline Streptoprocne zonaris & \multicolumn{2}{|l|}{ taperuçu-de-coleira-branca } \\
\hline Chaetura meridionalis & andorinhão-do-temporal & \\
\hline \multicolumn{3}{|l|}{ Trochilidae } \\
\hline Ramphodon naevius & beija-flor-rajado & \\
\hline Phaethornis squalidus & rabo-branco-pequeno & \\
\hline Phaethornis pretrei & rabo-branco-acanelado & \\
\hline Eupetomena macroura & beija-flor-tesoura & \\
\hline Aphantochroa cirrochloris & beija-flor-cinza & \\
\hline Florisuga fusca & beija-flor-preto & \\
\hline Anthracothorax nigricollis & beija-flor-de-veste-preta & \\
\hline Lophornis chalybeus & topetinho-verde & \\
\hline Chlorostilbon lucidus & \multicolumn{2}{|l|}{ besourinho-de-bico-vermelho } \\
\hline Thalurania glaucopis & beija-flor-de-fronte-violeta & \\
\hline Leucochloris albicollis & beija-flor-de-papo-branco & \\
\hline Amazilia versicolor & beija-flor-de-banda-branca & \\
\hline Amazilia fimbriata & \multicolumn{2}{|l|}{ beija-flor-de-garganta-verde } \\
\hline Clytolaema rubricauda & beija-flor-rubi & \\
\hline
\end{tabular}




\begin{tabular}{|c|c|c|}
\hline TROGONIFORMES & & \\
\hline Trogonidae & & \\
\hline Trogon viridis & $\begin{array}{l}\text { surucuá-grande-de-barriga- } \\
\text { amarela }\end{array}$ & EN \\
\hline Trogon surrucura & surucuá-variado & \\
\hline Trogon rufus & surucuá-de-barriga-amarela & \\
\hline CORACIIFORMES & & \\
\hline Alcedinidae & & \\
\hline Megaceryle torquata & martim-pescador-grande & \\
\hline Chloroceryle amazona & martim-pescador-verde & \\
\hline Chloroceryle americana & martim-pescador-pequeno & \\
\hline GALBULIFORMES & & \\
\hline Bucconidae & & \\
\hline Malacoptila striata & barbudo-rajado & \\
\hline PICIFORMES & & \\
\hline Ramphastidae & & \\
\hline Ramphastos vitellinus & tucano-de-bico-preto & \\
\hline Ramphastos dicolorus & tucano-de-bico-verde & \\
\hline Selenidera maculirostris & araçari-poca & \\
\hline Picidae & & \\
\hline Picumnus temminckii & pica-pau-anão-de-coleira & \\
\hline Picumnus nebulosus & pica-pau-anão-carijó & \\
\hline Melanerpes candidus & pica-pau-branco & \\
\hline Melanerpes flavifrons & benedito-de-testa-amarela & \\
\hline Veniliornis spilogaster & picapauzinho-verde-carijó & \\
\hline Piculus flavigula & pica-pau-bufador & $\mathrm{VU}$ \\
\hline Colaptes campestris & pica-pau-do-campo & \\
\hline Celeus flavescens & pica-pau-de-cabeça-amarela & \\
\hline Dryocopus lineatus & pica-pau-de-banda-branca & \\
\hline PASSERIFORMES & & \\
\hline Thamnophilidae & & \\
\hline Terenura maculata & zidedê & \\
\hline Myrmoderus squamosus & papa-formiga-de-grota & \\
\hline Rhopias gularis & choquinha-de-garganta-pintada & \\
\hline Myrmotherula unicolor & choquinha-cinzenta & \\
\hline Stymphalornis acutirostris & bicudinho-do-brejo & $\mathrm{CR}$ \\
\hline Dysithamnus stictothorax & choquinha-de-peito-pintado & \\
\hline Dysithamnus mentalis & choquinha-lisa & \\
\hline $\begin{array}{l}\text { Herpsilochmus } \\
\text { rufimarginatus }\end{array}$ & chorozinho-de-asa-vermelha & \\
\hline $\begin{array}{l}\text { Thamnophilus } \\
\text { caerulescens }\end{array}$ & choca-da-mata & \\
\hline Hypoedaleus guttatus & chocão-carijó & \\
\hline Batara cinerea & matracão & \\
\hline Mackenziaena leachii & borralhara-assobiadora & \\
\hline Drymophila rubricollis & trovoada-de-bertoni & \\
\hline Drymophila malura & choquinha-carijó & \\
\hline Drymophila squamata & pintadinho & EN \\
\hline Drymophila ferruginea & trovoada & \\
\hline Pyriglena leucoptera & papa-taoca-do-sul & \\
\hline Conopophagidae & & \\
\hline
\end{tabular}

Saúde Meio Ambient. v. 4, n. 2, p. 16-31, jul./dez. 2015 


\begin{tabular}{|c|c|c|}
\hline Conopophaga lineata & chupa-dente & \\
\hline Conopophaga melanops & \multirow{2}{*}{\multicolumn{2}{|c|}{ cuspidor-de-máscara-preta }} \\
\hline Grallariidae & & \\
\hline Grallaria varia & tovacuçu & \\
\hline Hylopezus nattereri & pinto-do-mato & \\
\hline \multicolumn{3}{|l|}{ Rhinocryptidae } \\
\hline Merulaxis ater & entufado & VU \\
\hline Eleoscytalopus indigoticus & macuquinho & \\
\hline Scytalopus speluncae & tapaculo-preto & \\
\hline \multicolumn{3}{|l|}{ Formicariidae } \\
\hline Formicarius colma & galinha-do-mato & \\
\hline Chamaeza campanisona & tovaca-campainha & \\
\hline Chamaeza ruficauda & tovaca-de-rabo-vermelho & \\
\hline \multicolumn{3}{|l|}{ Scleruridae } \\
\hline Sclerurus scansor & vira-folha & \\
\hline \multicolumn{3}{|l|}{ Dendrocolaptidae } \\
\hline Dendrocincla turdina & arapaçu-liso & \\
\hline Sittasomus griseicapillus & arapaçu-verde & \\
\hline Xiphorhynchus fuscus & arapaçu-rajado & \\
\hline $\begin{array}{l}\text { Campylorhamphus } \\
\text { falcularius }\end{array}$ & arapaçu-de-bico-torto & \\
\hline Lepidocolaptes falcinellus & arapaçu-escamado-do-sul & \\
\hline Dendrocolaptes platyrostris & arapaçu-grande & \\
\hline Xiphocolaptes albicollis & \multicolumn{2}{|l|}{ arapaçu-de-garganta-branca } \\
\hline \multicolumn{3}{|l|}{ Furnariidae } \\
\hline Xenops minutus & bico-virado-miúdo & \\
\hline Lochmias nematura & joão-porca & \\
\hline Automolus leucophthalmus & \multicolumn{2}{|l|}{ barranqueiro-de-olho-branco } \\
\hline Anabazenops fuscus & trepador-coleira & \\
\hline Anabazenops lichtensteini & limpa-folha-ocráceo & \\
\hline Philydor atricapillus & limpa-folha-coroado & \\
\hline Philydor rufum & limpa-folha-de-testa-baia & \\
\hline Heliobletus contaminatus & trepadorzinho & \\
\hline Anabacerthia amaurotis & limpa-folha-miúdo & \\
\hline Syndactyla rufosuperciliata & trepador-quiete & \\
\hline Cichlocolaptes leucophrus & trepador-sobrancelha & \\
\hline Leptasthenura setaria & grimpeiro & \\
\hline Certhiaxis cinnamomeus & curutié & \\
\hline Synallaxis ruficapilla & pichororé & \\
\hline Synallaxis cinerascens & pi-puí & \\
\hline Synallaxis spixi & joão-teneném & \\
\hline Cranioleuca obsoleta & arredio-oliváceo & \\
\hline Cranioleuca pallida & arredio-pálido & \\
\hline \multicolumn{3}{|l|}{ Pipridae } \\
\hline Manacus manacus & rendeira & \\
\hline Ilicura militaris & tangarazinho & \\
\hline Chiroxiphia caudata & tangará & \\
\hline \multicolumn{3}{|l|}{ Onychorhynchidae } \\
\hline Myiobius barbatus & assanhadinho & EN \\
\hline \multicolumn{3}{|l|}{ Tityridae } \\
\hline Schiffornis virescens & flautim & \\
\hline
\end{tabular}




\begin{tabular}{|c|c|c|}
\hline Tityra cayana & anambé-branco-de-rabo-preto & \\
\hline Pachyramphus castaneus & caneleiro & \\
\hline $\begin{array}{l}\text { Pachyramphus } \\
\text { polychopterus }\end{array}$ & caneleiro-preto & \\
\hline Pachyramphus validus & caneleiro-de-chapéu-preto & \\
\hline \multicolumn{3}{|l|}{ Cotingidae } \\
\hline Procnias nudicollis & araponga & \\
\hline Pyroderus scutatus & pavó & EN \\
\hline Carpornis cucullata & corocochó & \\
\hline \multicolumn{3}{|l|}{ Platyrinchidae } \\
\hline Platyrinchus mystaceus & patinho & \\
\hline Platyrinchus leucoryphus & patinho-gigante & VU \\
\hline \multicolumn{3}{|l|}{ Rhynchocyclidae } \\
\hline Mionectes rufiventris & abre-asa-de-cabeça-cinza & \\
\hline $\begin{array}{l}\text { Leptopogon } \\
\text { amaurocephalus }\end{array}$ & cabeçudo & \\
\hline Phylloscartes eximius & barbudinho & $\mathrm{CR}$ \\
\hline Phylloscartes ventralis & borboletinha-do-mato & \\
\hline Phylloscartes kronei & maria-da-restinga & \\
\hline Phylloscartes paulista & não-pode-parar & \\
\hline Phylloscartes oustaleti & papa-moscas-de-olheiras & VU \\
\hline Phylloscartes difficilis & estalinho & EN \\
\hline Phylloscartes sylviolus & maria-pequena & EN \\
\hline Tolmomyias sulphurescens & bico-chato-de-orelha-preta & \\
\hline Todirostrum poliocephalum & teque-teque & \\
\hline Poecilotriccus plumbeiceps & tororó & \\
\hline Myiornis auricularis & miudinho & \\
\hline Hemitriccus obsoletus & catraca & \\
\hline Hemitriccus orbitatus & tiririzinho-do-mato & \\
\hline Hemitriccus kaempferi & maria-catarinense & VU \\
\hline \multicolumn{3}{|l|}{ Tyrannidae } \\
\hline Camptostoma obsoletum & risadinha & \\
\hline Elaenia flavogaster & \multicolumn{2}{|l|}{ guaracava-de-barriga-amarela } \\
\hline Elaenia parvirostris & guaracava-de-bico-curto & \\
\hline Elaenia mesoleuca & tuque & \\
\hline Elaenia obscura & tucão & \\
\hline Myiopagis caniceps & guaracava-cinzenta & \\
\hline Phyllomyias virescens & piolhinho-verdoso & \\
\hline Phyllomyias fasciatus & piolhinho & \\
\hline Serpophaga subcristata & alegrinho & \\
\hline Attila phoenicurus & capitão-castanho & \\
\hline Attila rufus & capitão-de-saíra & \\
\hline $\begin{array}{l}\text { Ramphotrigon } \\
\text { megacephalum }\end{array}$ & maria-cabeçuda & \\
\hline Myiarchus swainsoni & irré & \\
\hline Myiarchus ferox & maria-cavaleira & \\
\hline Sirystes sibilator & gritador & \\
\hline Machetornis rixosa & suiriri-cavaleiro & \\
\hline Myiodynastes maculatus & bem-te-vi-rajado & \\
\hline Myiozetetes similis & bentevizinho-de-penacho-vermelho & \\
\hline Tyrannus melancholicus & suiriri & \\
\hline
\end{tabular}

Saúde Meio Ambient. v. 4, n. 2, p. 16-31, jul./dez. 2015 


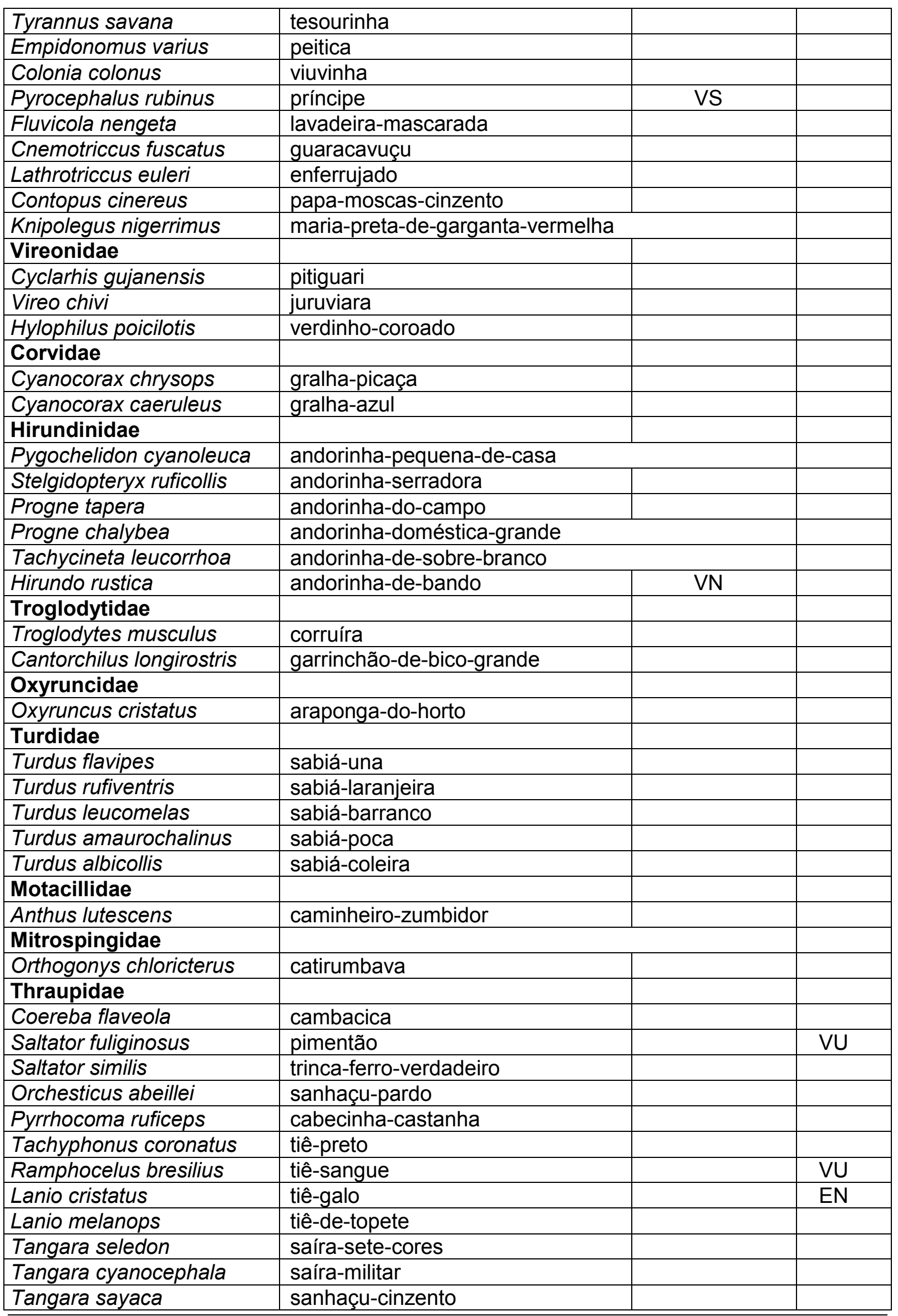




\begin{tabular}{|c|c|c|}
\hline Tangara cyanoptera & sanhaçu-de-encontro-azul & \\
\hline Tangara palmarum & sanhaçu-do-coqueiro & \\
\hline Tangara ornata & sanhaçu-de-encontro-amarelo & \\
\hline Tangara peruviana & saíra-sapucaia & EN \\
\hline Tangara preciosa & saíra-preciosa & \\
\hline $\begin{array}{l}\text { Stephanophorus } \\
\text { diadematus }\end{array}$ & sanhaçu-frade & \\
\hline Pipraeidea melanonota & saíra-viúva & \\
\hline Tersina viridis & saí-andorinha & \\
\hline Dacnis nigripes & saí-de-pernas-pretas & \\
\hline Dacnis cayana & saí-azul & \\
\hline Chlorophanes spiza & saí-verde & \\
\hline Hemithraupis ruficapilla & saíra-ferrugem & \\
\hline Conirostrum bicolor & figuinha-do-mangue & VU \\
\hline Haplospiza unicolor & cigarra-bambu & \\
\hline Poospiza thoracica & peito-pinhão & \\
\hline Poospiza cabanisi & tico-tico-da-taquara & \\
\hline Volatinia jacarina & Tiziu & \\
\hline Sporophila frontalis & Pixoxó & VU \\
\hline Sporophila lineola & Bigodinho & \\
\hline Sporophila caerulescens & Coleirinho & \\
\hline Sporophila angolensis & Curió & CR \\
\hline Tiaris fuliginosus & cigarra-do-coqueiro & \\
\hline \multicolumn{3}{|l|}{ Passerellidae } \\
\hline Zonotrichia capensis & tico-tico & \\
\hline \multicolumn{3}{|l|}{ Cardinalidae } \\
\hline Habia rubica & tiê-do-mato-grosso & \\
\hline \multicolumn{3}{|l|}{ Parulidae } \\
\hline Setophaga pitiayumi & Mariquita & \\
\hline Geothlypis aequinoctialis & pia-cobra & \\
\hline Basileuterus culicivorus & pula-pula & \\
\hline $\begin{array}{l}\text { Basileuterus } \\
\text { leucoblepharus }\end{array}$ & pula-pula-assobiador & \\
\hline Phaeothlypis rivularis & pula-pula-ribeirinho & \\
\hline \multicolumn{3}{|l|}{ Icteridae } \\
\hline Cacicus chrysopterus & Tecelão & \\
\hline Cacicus haemorrhous & Guaxe & \\
\hline Chrysomus ruficapillus & Garibaldi & \\
\hline Agelaioides badius & asa-de-telha & \\
\hline Molothrus bonariensis & vira-bosta & \\
\hline Sturnella superciliaris & polícia-inglesa-do-sul & \\
\hline \multicolumn{3}{|l|}{ Fringillidae } \\
\hline Sporagra magellanica & Pintassilgo & \\
\hline Euphonia chlorotica & fim-fim & \\
\hline Euphonia violacea & gaturamo-verdadeiro & \\
\hline Euphonia chalybea & cais-cais & \\
\hline Euphonia cyanocephala & gaturamo-rei & \\
\hline Euphonia pectoralis & ferro-velho & \\
\hline \multicolumn{3}{|l|}{ Estrildidae } \\
\hline Estrilda astrild & bico-de-lacre & \\
\hline Passeridae & & \\
\hline
\end{tabular}




\begin{tabular}{l|l|l|}
\hline Passer domesticu & pardal & \\
\hline Legenda: CR - Criticamente em Perigo; En - Em Perigo; Vu - Vulnerável; VS - Visitantes do \\
Hemisfério Sul; VN - Visitantes do Hemisfério Norte.
\end{tabular}

Dentre as espécies levantadas, 31 constam na lista de espécies ameaçadas de extinção para o estado de Santa Catarina (CONSEMA, 2011), dentre as quais 15 são categorizadas como vulneráveis, 11 em perido e 5 criticamente em perígo, indicando a importância do município na preservação destas espécies.

No que diz respeito às aves migratórias, foi cadastrado para o município 18 espécies (15 Visitantes do Hemisfério Norte e 3 Visitantes do Hemisfério Sul), representando $14 \%$ das 172 espécies registradas para o Brasil, conforme a lista das espécies de aves migratórias ocorrentes no Brasil, (CEMAVE, 2005).

\section{CONSIDERAÇÕES FINAIS}

A riqueza de espécies levantadas para o município de Joinville pode ser considerada expressiva, estando esta relacionada com o significativo esforço amostral empregado durante as campanhas de campo, assim como a diversidade de ambientes ocorrentes no município. A sazonalidade dos levantamentos e as várias metodologias aplicadas também contribuíram para este resultado positivo, maximizando as chances de detecção de um maior número de espécies.

Apesar da elevada riqueza de espécies obtidas, é importante a continuidade dos estudos ornitológicos no município, principalmente os relacionados a abundância das espécies ameaçadas, buscando estabelecer medidas conservacionistas para o município.

\section{REFERÊNCIAS}

AMORIM, J.F.; PIACENTINI, V.Q. Novos registros de aves raras em Santa Catarina, Sul do Brasil, incluindo os primeiros registros documentados de algumas espécies para o Estado. Revista Brasileira de Ornitologia, v. 14, n. 2, p. 145-149, 2006.

AZEVEDO, M.A.G.; I.R. GHIZONI-JR. Novos registros de aves para o estado de Santa Catarina, sul do Brasil. Atualidades Ornitológicas, n. 126, p. 9-12, 2005.

CENTRO NACIONAL DE PESQUISA PARA CONSERVAÇÃO DAS AVES SILVESTRES. Lista das Espécies de Aves Migratórias Ocorrentes no Brasil. 2005. Disponível em: <www.ibama.gov.br/cemave>. Acesso em: 08 set. 2014. 
COMITÊ BRASILEIRO DE REGISTROS ORNITOLÓGICOS. Listas das aves do Brasil, 2014. Disponível em <http://www.cbro.org.br>. Acesso em: 06 ago. 2014.

CONSELHO ESTADUAL DO MEIO AMBIENTE. Resolução Consema $\mathbf{n}^{\circ}$ 02/2011. Reconhece a Lista Oficial de Espécies da Fauna Ameaçadas de Extinção no Estado de Santa Catarina e dá outras providências.

CREMER, M. J.; GROSE, A. V. Aves do Estuário da Babitonga e Litoral de São Francisco do Sul. Joinville: Editora UNIVILLE, 2010. 192 p.

GHIZONI-JUNIOR. I.R. Registro de Polioptila dumicola (Aves: Muscicapidae, Sylviinae) no estado de Santa Catarina, sul do Brasil. Biotemas, v. 17, n. 2, p. 205208, 2004.

GHIZONI-JR. I. R.; SILVA, E. S. da. Registro do saí-canário Thlypopsis sórdida (D'Orbigny \& Lafresnaye, 1837) (Aves, Thraupidae) no Estado de Santa Catarina, sul do Brasil. Biotemas, v. 19, n. 2, p. 81-82, 2006.

GHIZONI-JR. I.R. et al. Registro da pomba-do-orvalho Pata-gioenas maculosa (Aves: Columbidae) no estado de Santa Catarina, sul do Brasil. Biotemas, v. 22, n.2, p. 195-197, 2009.

GHIZONI-JR et al. Checklist da avifauna da Ilha de Santa Catarina, sul do Brasil. Atualidades Ornitológicas, n. 171, 2013. Disponível em:

<http://www.ao.com.br/download/AO171_50.pdf>. Acesso em: 22 out. 2014.

GROSE, A. V. et al. Aves Migratórias no Estuário da Baía da Babitonga e Litoral de São Francisco do Sul, Litoral Sul do Brasil. In: XIV Congresso Latino-Americano de Ciências do Mar - XIV COLACMAR, 2011, Balneário Camboriú.

GROSE, A. V. Avifauna em três unidades de conservação urbanas no município de Joinville, Santa Catarina, Brasil. Atualidades Ornitológicas n. 175, 2013.

INSTITUTO BRASILEIRO DE GEOGRAFIA E ESTATÍSTICA. Disponível em <http://cidades.ibge.gov.br/painel/painel.php?codmun=420910> Acesso em: 19 mar. 2015.

INSTITUTO BRASILEIRO DO MEIO AMBIENTE E DOS RECURSOS NATURAIS RENOVÁVEIS. Lista nacional da fauna brasileira ameaçada de extinção, 2003. Disponível <http://www.mma.gov.br/port/sbf/fauna/index.cfm>. Acesso em: 08 ago. 2014.

MOREIRA-LIMA, L.M. 2013. Aves da mata atlântica: riqueza, composição, status, endemismos e conservação. São Paulo: Instituto de biociências, universidade de São Paulo, 2013. Dissertação de mestrado em zoologia. Disponível em: $<$ http://w.teses.usp.br/teses/disponiveis/41/41133/tde-17042014-091547/>. Acesso em: 25 out. 2014. 
NUNES, A. P.; TOMAS W. M. Aves migratórias e nômades ocorrentes no Pantanal. Corumbá: Embrapa Pantanal, 2008. Disponível em:

<http://www.cpap.embrapa.br/publicacoes/online/DOC62.pdf>. Acesso em: 22 mar. 2015

RUPP, A. E. et al. Novas espécies de aves para Santa Catarina, sul do Brasil. Biotemas, v. 21, n. 3 p. 163-168, 2008.

SEVEGNANI, G. C. Avifauna no fragmento florestal do Jardim Botânico da Univille e em seu entorno. Revista Univille, v. 14, n. 2 p. 25-32, 2009.

SICK, H. Ornitologia brasileira. Rio de Janeiro: Nova Fronteira, 1997.

SIGRIST, T. Aves do Brasil: uma visão artística. São Paulo: Fosfertil, 672p, 2006.

SOUZA, D. Todas as aves do Brasil: guia de campo para identificação. 2.ed. Feira de Santana: Dall, 2004.

WEIMER, C. O. et al. Levantamento da avifauna em um fragmento florestal localizado no centro urbano do município de xanxerê, SC. Unoesc \& Ciência ACBS, v. 5, n. 1, p. 91-102, 2014.

Artigo recebido em: 15/12/2014

Artigo aprovado em: 05/10/2015 